\title{
Transcription factor regulatory network for early lung immune response to tuberculosis in mice
}

\author{
MOEIN YAQUBI $^{1 *}$, ABDULSHAKOUR MOHAMMADNIA $^{2 *}$ and HOSSEIN FALLAHI ${ }^{3,4}$ \\ Departments of ${ }^{1}$ Medical Biotechnology and ${ }^{2}$ Molecular Biotechnology, \\ National Institute of Genetic Engineering and Biotechnology, Tehran 14178-63171; ${ }^{3}$ Medical Biology Research Center, \\ Kermanshah University of Medical Sciences, Kermanshah 67149-67346; ${ }^{4}$ Department of Biology, School of Science, \\ Razi University, Kermanshah 67149-67346, Iran
}

Received July 1, 2014; Accepted March 23, 2015

DOI: $10.3892 / \mathrm{mmr} .2015 .3721$

\begin{abstract}
Numerous transcription factors (TFs) have been suggested to have a role in Mycobacterium tuberculosis infection; however, the TFs involved in the early immune response of lung cells remains to be fully elucidated. The present study aimed to identify TFs which may have a role in the early immune response to tuberculosis and the gene regulatory networks in which they are involved. Gene expression data obtained from microarray analysis of the early lung immune response to tuberculosis (Gene Expression Omnibus; accession no. GSE23014) was integrated with data for TF binding sites and protein-protein interactions in order to construct a TF regulatory network. The role of TFs in protein complexes, active modules, topology of the network and regulation of immune processes were investigated. The results demonstrated that the constructed gene regulatory network harbored 1,270 differentially expressed (DE) genes with 4,070 regulatory and protein-protein interactions. In addition, it was revealed that 17 DE TFs were involved in the positive regulation of numerous immunological and biological processes, including $\mathrm{T}$ cell activation, $\mathrm{T}$ cell proliferation and tuberculosis-associated gene expression, in the constructed regulatory network. Signal transducer and activator of transcription 4, interferon regulatory factor 8 , spleen focus-forming virus proviral integration 1, enhancer of zeste homolog 2 and kruppel-like factor 4 were predicted to be the primary TFs regulating the DE genes during early lung infection by $M$. tuberculosis, as determined through various analyses of the gene regulatory network. In conclusion, the present study identified novel TFs
\end{abstract}

Correspondence to: Dr Hossein Fallahi, Department of Biology, School of Science, Razi University, Bagh-e Abrisham, Kermanshah 67149-67346, Iran

E-mail: h.fallahi@razi.ac.ir

*Contributed equally

Key words: early lung infection, gene regulatory network, immune response, transcription factor, tuberculosis involved in the early response to M. tuberculosis infection, which may enhance current understanding of the molecular mechanism underlying tuberculosis infection and introduced potential targets for novel tuberculosis therapies.

\section{Introduction}

Tuberculosis is a disease caused by the intracellular bacterium Mycobacterium tuberculosis, which has a high mortality rate (1). $\mathrm{CD}^{+} \mathrm{T}$ lymphocytes have been reported to have a central role in the defense against $M$. tuberculosis and $\mathrm{T}$ helper 1-type (Th1) T cell-mediated immunity is the primary response during tuberculosis (2-4). A previous study reported that $M$. tuberculosis infection in the absence of $\alpha-\beta$ T lymphocytes caused mortality in mice within 48 days (5). Protective immunity against $M$. tuberculosis is predominantly regulated by transcription factors (TFs).

Numerous TFs have been reported to be involved in the immune response to tuberculosis. Protective immunity against $M$. tuberculosis infection was reported to be under regulation of the TF signal transducer and activator of transcription (Stat) 4 (6). Nuclear factor of activated T cells p (NFATp) and tumor necrosis factor (TNF) were suggested to regulate the inflammatory response, while mice deficient for NFATp had an increased mortality rate following the development of tuberculosis (7). BXH2 mice deficient for the $\operatorname{Irf} 8^{294 c}$ allele succumbed to the disease due to uncontrolled growth of M. tuberculosis (8). Expression of spleen focus-forming virus proviral integration 1 (Sfpi1) TF was demonstrated to be increased following 28 days of M. tuberculosis infection (9). Significant overlap between the binding sites of interferon regulatory factor 8 (Irf8) and Sfpil was observed using chromatin immunoprecipitation microarray (ChIP-chip) analysis of tuberculosis infection (10). Th1 cells have a crucial role in defense against tuberculosis. V-rel avian reticuloendotheliosis viral oncogene homolog (Rel) B (RelB) TF is essential for differentiation of Th1 cells and its deficiency was reported to induce defects in the differentiation of these cells (11). In addition to RelB, Stat4 and Stat1 were demonstrated to be involved in the development of Th1 cells in response to infection (12). Therefore, investigating these individual TFs may elucidate numerous underlying processes in tuberculosis; however, it 
may not provide an overview of gene regulation during tuberculosis.

Construction of gene regulatory networks in tuberculosis, which include genes and TFs, provide a novel opportunity for understanding the dynamic of molecular processes involved in the disease. Such networks have been constructed for M. tuberculosis. A previous study reviewed and constructed a gene regulatory network for $M$. tuberculosis genes involved in persistence in order to provide insight into the molecular mechanisms involved in persistency (13). In addition, a comprehensive network of infection-associated processes in human macrophages following $M$. tuberculosis infection was constructed (14) as well as a host intracellular network for the regulation of $M$. tuberculosis survival (15). However, there has not yet been a regulatory network constructed for the TFs involved in the early lung immune response to M.tuberculosis infection.

In the present study, a network was constructed of the genes that were differentially expressed (DE) specifically in the lung cells in response to tuberculosis. TF binding sites, protein-protein interactions and expression data were integrated in order to construct the gene regulatory network. Network analyses using system biology tools were used to determine the most prominent TFs involved in early lung immune responses to tuberculosis.

\section{Materials and methods}

Microarray availability and preprocessing. Raw data for early lung infection with $M$. tuberculosis were obtained from the Gene Expression Omnibus (GEO; http://www.ncbi.nlm.nih. gov/geo/) server database (accession no. GSE23014). These data contributed by Kang et al (16), contained time course ( 0 , 12,15 and 21 days) expression data for infection of C57BL/6 mice with the H37Rv strain of M. tuberculosis. Microarray samples were divided into three groups for the comparison and identification of DE genes during the early days of lung response to tuberculosis: Group 1, data from 12 days post infection was compared with that of day 0 ; group 2, comparison of days 12 and 15 post infection; and group 3, comparison of day 15 with day 21 post infection.

A Robust Multi-array Averaging (RMA) algorithm was used for normalization of raw data (17). DE genes were identified using a two-sample Student's t-test algorithm. These algorithms were each performed using Flexarray software v1.6.2 (18). A fold change of 1.5 was set as the threshold criteria for identifying DE genes. Group III data was used for all subsequent analysis.

Functional clustering DE genes. In order to determine the enrichment process during each comparison, the Databases for Annotation, Visualization and Integrated Discovery (DAVID; http://david.abcc.ncifcrf.gov/) bioinformatics tool was used. Clusters with enrichment scores of $>1.3$ were regarded to be meaningful clusters in the functional clustering analysis (19).

TFs involved in regulating DE genes. In order to distinguish regulators of DE genes in each comparison, data was submitted to the ChIP Enrichment Analysis (ChEA; http://amp.pharm.mssm.edu/lib/chea.jsp) database (20). This database analyzed 458,471 potential regulatory interactions based on ChEA from 221 publications. In addition, the TFactS database (http://www.tfacts.org/) was used, which integrates data from experimentally validated TFs/targets regulatory interactions. This database consists of data from 6,401 regulatory interactions for $343 \mathrm{TFs}$ which regulate 2,720 genes from the following databases: PAZAR, Transcriptional Regulatory Element Database, Nuclear Factor I Regulome Database and Transcription Regulatory Regions Database, as well as literature. TFactS was used to compare submitted DE gene data from the present study with validated target genes available in its catalog in order to identify the regulatory genes in the submitted datasets (21). TFs were limited based on their P-value $(<0.05)$ and altered expression $(\geq 1.5$ fold change).

TFs protein-protein interactions. The Biological General Repository for Interaction Datasets (BioGRID; http://thebiogrid. $\mathrm{org} /$ ) database was used to extract valid protein-protein interactions information for DE TFs (22). In addition to BioGRID, protein-protein interaction data was obtained from the Search Tool for the Retrieval of Interacting Genes/Proteins (STRING; http://string-db.org/) database, which accommodates physical (validated and predicted) and functional interactions. Interactions with a confidence score $>0.4$ (medium confidence) were incorporated into the networks (23).

The protein interactions obtained from BioGRID and STRING were mapped to the expression data in order to identify only meaningful interactions. These interactions contributed to the construction of the TFs regulatory network and TFs protein-protein interaction network.

Network construction and ontology. In order to construct regulatory networks, TF/target interactions, TFs protein-protein interactions and expression data were integrated and visualized using Cytoscape v3.0.2 (24). The enriched immune system processes were investigated in the constructed network using the ClueGO v1.8 plugin for Cytoscape. A two-sided hypergeometric statistical test and Bonferroni correction were used for P-value correction. In addition, ClueGO was used to determine processes affected by the DE TFs based on Kyoto Encyclopedia of Genes and Genomes (KEGG; http://www.genome.jp/kegg/) pathway and biological process analyses (25).

Centrality analysis of regulatory network. Central genes in the regulatory network were identified using a CentiScaPe v2.0 plugin for Cytoscape. Three centrality indexes were used on a directed regulatory network: Degree, betweenness and stress. Degree is considered to be the simplest index for topological analysis; the number of adjacent connected nodes to a given node (x) reveal the degree of this node. In the directed network constructed, out-degree rather than in-degree was considered. Nodes with a high degree are considered the hubs of the network. In addition to degree, stress and betweenness were used as centrality indexes to find hub TFs. These two indexes provide complementary results from analysis of the central genes (26).

Protein complexes and active modules. Protein-protein interactions obtained for DE TFs from BioGRID and STRING were used to construct a protein interaction network. This network was used to identify protein complexes which may 
be involve in the response of lung cells to early infection of tuberculosis. These protein complexes were identified using MCODE v1.4.1 plugin for Cytoscape (27).

Integrated regulatory networks were composed of expression data and interactions. Parts of this network demonstrated increased activity compared with other parts based on expression, these are referred to as active modules. These modules were explored in Cytoscape using the JActiveModules v1.8 tool (28). To identify active modules in constructed regulatory networks using JActiveModules, the expression values were converted to P-values. JActiveModules find active modules using the loaded P-values.

Summary of methods. Overall, the most crucial DE TFs involve in early lung immune response to tuberculosis were identified based on several analyses, including presence in protein complexes, contribution in active modules, centrality as well as role in regulation of significant processes and tuberculosis-associated genes. These analyses were divided into three parts: Involvement in protein complexes and active modules; centrality analysis; and regulation of the most enriched processes. DE TFs that were present in $\geq 2$ out of 3 of these analyses were assumed to be important factors in early lung immune response to tuberculosis.

\section{Results}

Gene expression during different stages of early lung responses to tuberculosis. Gene expression data obtained from early lung immune response to tuberculosis was divided into three distinct groups. By comparing the expression profiles at day 0 and 12 post infection (Group I), $240 \mathrm{DE}$ genes were identified, while 153 and 2,105 DE genes were detected when the gene expression profiles were compared between day 12 and 15 (Group II) and between day 15 and 21 (Group III), respectively. As the number of DE genes obtained from group III was higher than those of the two other groups, the third comparison was selected to establish the regulatory network of TFs in lungs during infection by M. tuberculosis.

Functional clustering analysis of DE genes obtained from the comparison of day 15 and 21 post M. tuberculosis infection resulted in the identification of 113 meaningful clusters with an accepted enrichment score of $>1.3$. The presence of immune system-associated terms, including those for signaling, defense response, inflammatory response and $\mathrm{T}$ cell activation, indicated the involvement of the immune system during the early response of lung cells infected by $M$. tuberculosis.

Regulators of DE genes. ChEA and TFactS databases revealed the involvement of $17 \mathrm{DE}$ TFs in the regulation of early responses, including eomesodermin, v-ets avian erythroblastosis virus E26 oncogene homolog, enhancer of zeste homolog 2 (Ezh2), Irf8, jun B proto-oncogene, kruppel-like factor 4 (Klf4), myeloblastosis-related protein B (Mybl2), nuclear receptor subfamily 3 group C member 1, Rel, RelB, Sfpi1, sex determining region Y-box 17, Stat1, Stat2, Stat4, T-cell acute lymphocytic leukemia 1 (Tal1) and thyrotroph embryonic factor. These DE TFs contribute to the regulation of 1,253 out of 2,105 DE genes; however, TFs were

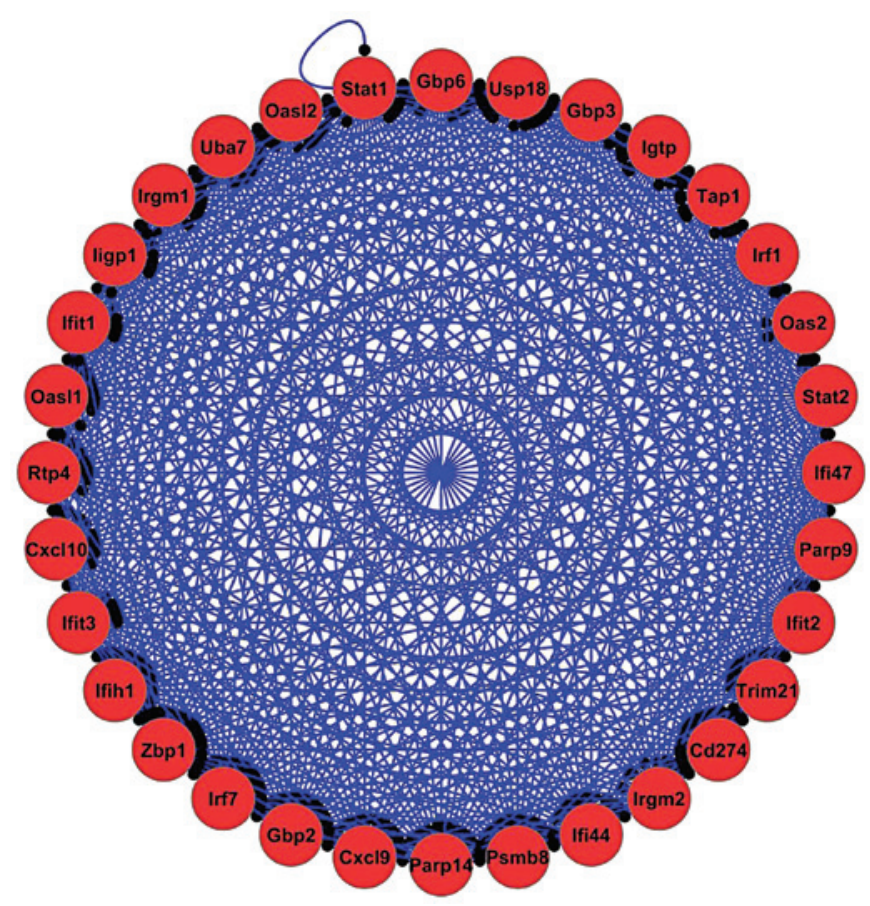

Figure 1. Protein complex with highest score in the TFs protein-protein interaction network. Red nodes represent upregulated proteins and blue lines indicate protein-protein interactions. Black dots indicate the direction of interactions.

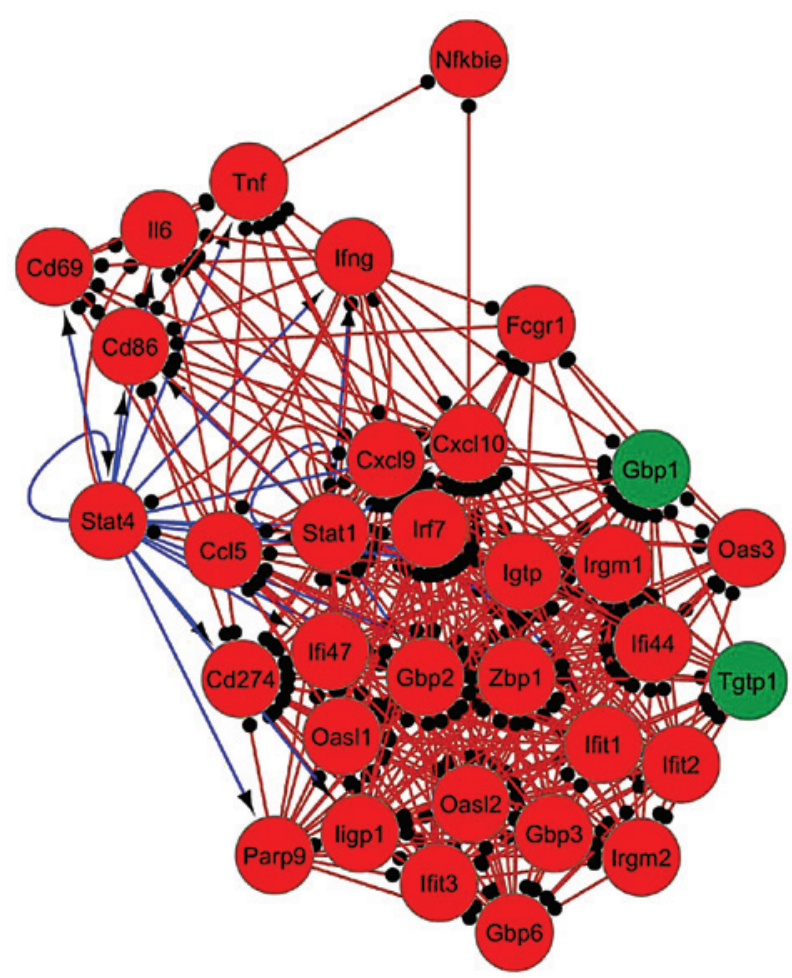

Figure 2. Top active modules extracted from the gene regulatory network. Red and green nodes indicate up- and downregulated modules, respectively. Blue and red lines represent protein-DNA and protein-protein interactions, respectively. Arrows and dots indicate the direction of protein-DNA and protein-protein interactions, respectively.

not identified for the remainder of the DE genes submitted. Following protein-protein interaction analysis of these TFs, a gene regulatory network was constructed, including all 
Table I. Ranking of TFs based on analyses of three centrality indexes.

\begin{tabular}{|c|c|c|c|c|c|c|}
\hline \multirow[b]{2}{*}{ Symbol } & \multirow[b]{2}{*}{ TF name } & \multicolumn{3}{|c|}{ Centrality indexes } & \multirow[b]{2}{*}{ Mean } & \multirow[b]{2}{*}{ Rank } \\
\hline & & Degree & Stress & Betweenness & & \\
\hline Sfpi1 & $\begin{array}{l}\text { Spleen focus-forming virus } \\
\text { proviral integration } 1\end{array}$ & 1 & 1 & 1 & 1.00 & 1 \\
\hline Stat4 & Stat4 & 5 & 2 & 2 & 3.00 & 2 \\
\hline Ezh2 & Enhancer of zeste homolog 2 & 4 & 3 & 4 & 3.66 & 3 \\
\hline Mybl2 & Myeloblastosis-related protein B & 7 & 4 & 3 & 4.66 & 4 \\
\hline Irf8 & Interferon regulatory factor 8 & 8 & 5 & 5 & 6.00 & 5 \\
\hline Sox 17 & Sex determining region Y-box 17 & 6 & 7 & 8 & 7.00 & 6 \\
\hline Eomes & Eomesodermin & 10 & 8 & 6 & 8.00 & 7 \\
\hline Stat1 & Stat1 & 12 & 6 & 7 & 8.33 & 8 \\
\hline Klf4 & Kruppel-like factor 4 & 2 & 13 & 13 & 9.33 & 9 \\
\hline $\mathrm{Nr} 3 \mathrm{c} 1$ & $\begin{array}{l}\text { Nuclear receptor subfamily } 3 \\
\text { group C member } 1\end{array}$ & 11 & 9 & 9 & 9.66 & 10 \\
\hline Stat2 & Stat2 & 13 & 11 & 11 & 11.66 & 11 \\
\hline Junb & Jun B proto-oncogene & 15 & 10 & 10 & 11.66 & 11 \\
\hline Tal1 & T-cell acute lymphocytic leukemia 1 & 3 & 17 & 17 & 12.33 & 12 \\
\hline Rel & Rel & 14 & 12 & 12 & 12.66 & 13 \\
\hline Erg & $\begin{array}{l}\text { V-ets avian erythroblastosis virus } \\
\text { E26 oncogene homolog }\end{array}$ & 9 & 16 & 16 & 13.66 & 14 \\
\hline RelB & RelB & 15 & 14 & 14 & 14.33 & 15 \\
\hline Tef & Thyrotroph embryonic factor & 16 & 15 & 15 & 15.33 & 16 \\
\hline
\end{tabular}

TF, transcription factor; Stat, signal transducer and activator of transcription; Rel, v-rel avian reticuloendotheliosis viral oncogene homolog.

seventeen TFs and 1,270 DE genes, which were revealed to be connected by 4070 interactions.

TFs involved in protein complexes and modules. In order to determine the involvement of TFs in protein complexes, the MCODE tool for Cytoscape was used. A total of 6 complexes were identified in early lung immune response to tuberculosis with a score of $\geq 3$. The top protein complex (score 30.516 based on the reference of MCODE plugin of Cytoscape) contained 32 DE genes with 474 linking interactions (Fig. 1). Biological process analysis of this protein complex indicated that the innate immune response was the top term, as determined using P-values and the number of DE genes. The DE TFs Ezh2, Irf8, Klf4, Rel, RelB, Stat1 and Stat2 were identified to be present in the protein complexes identifies.

The active modules of the regulatory network were analyzed in order to provide an in-depth view of the activity of the network's components. TFs involved in these modules are likely to be more important compared with other TFs. Based on the biological process analysis of the top active modules, the biological processes which were most affected by these TFs were as follows: Response to cytokine stimulus, response to viruses and the innate immune response. The top active module was composed of $33 \mathrm{DE}$ genes with 348 interactions (Fig. 2); DE genes in this module were primarily regulated by the TFs Stat 4 and Stat1. The top 5 modules were investigated for the presence of DE TFs and the results revealed that the TFs Stat4, Stat2, Stat1, Rel and RelB were detected in $\geq 1$ of the these modules. Of note, Stat 4 and Stat 1 were present in all of 5 modules. These results therefore demonstrated the central role of Stat 4 and Stat1 in active modules in the early lung response to tuberculosis infection. Overall, the TFs Ezh2, Irf8, Klf4, Rel, RelB, Stat1, Stat2 and Stat4 were identified to be involved in protein complexes and core active modules.

Central DE TFs in the regulatory network. Centrality analysis using the CentiScaPe plugin in Cytoscape highlighted the importance of TFs in the network topology and regulation of the whole network (Table I). Three parameters were considered in analyzing the network: Degree, betweenness and stress. According to degree, Sfpi1, Klf4, Tal1, Ezh2 and Stat4 were the DE TFs with highest interactions in the constructed gene regulatory network during early infection of the lung with tuberculosis. Rankings for stress and betweenness revealed that Sfpi1, Stat4, Ezh2, Mybl2 and Irf8 were the top 5 central genes in gene regulatory network. The overall ranking of the DE TFs, according to the mean ranking of the three parameters, identified Sfpi1, Stat4, Ezh2, Mybl2 and Irf8 as the top regulatory TFs in the constructed gene regulatory network.

TFs involvement in the regulation of immune system processes. In order to determine the most important TFs in the regulation of immune system processes, gene regulatory network ontology was used to identify the affected immune processes and their regulators (Table II). According to the P-values of affected processes in immune system analysis, the results demonstrated 


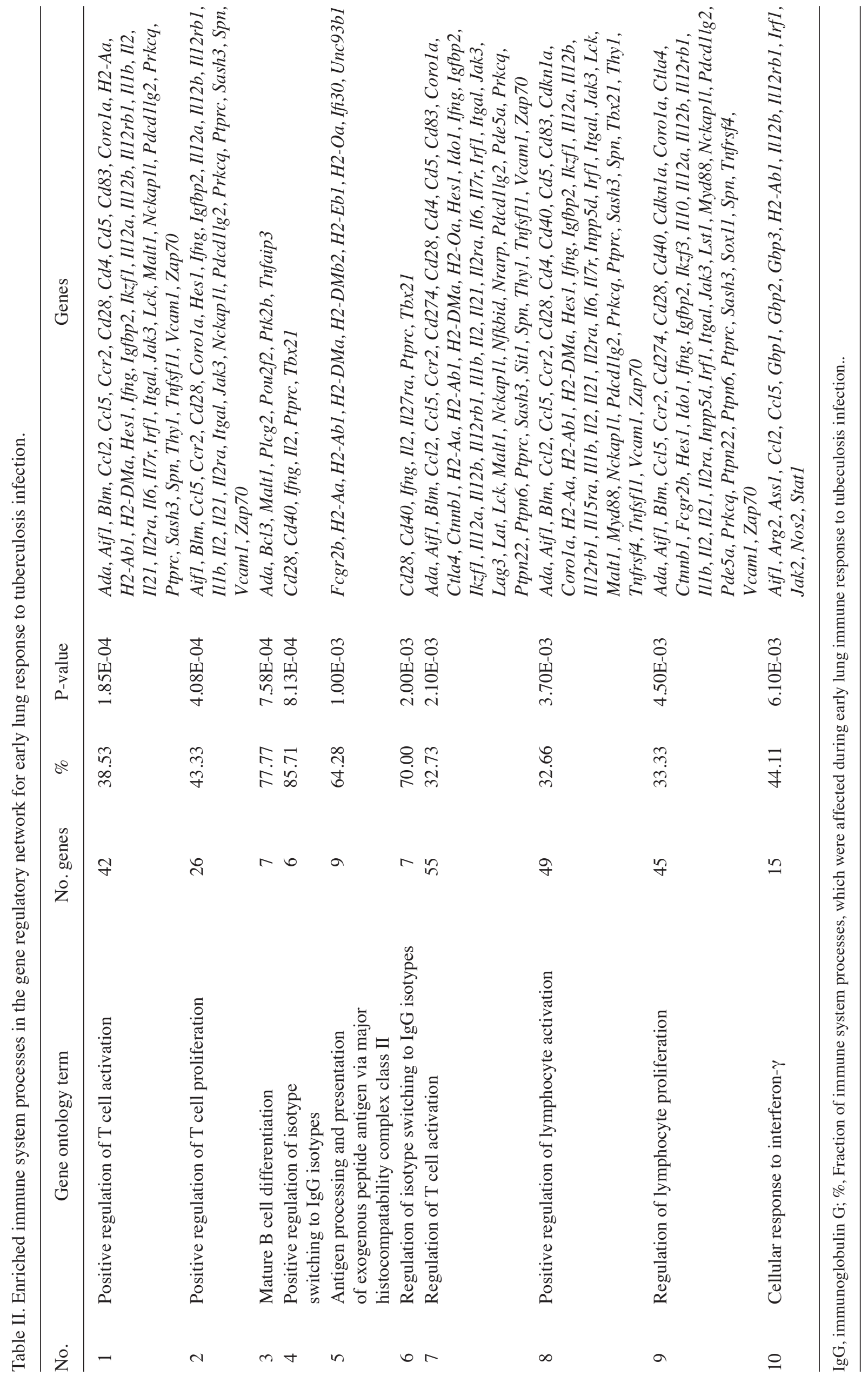


that the positive regulation of $\mathrm{T}$ cell activation and proliferation were the most enriched process. In the constructed regulatory gene network the most important regulatory DE TFs (based on number of regulatory interactions) for $\mathrm{T}$ cell activation included Sfpi1, Stat4. Tal1, Rel and Klf4. Positive regulation of T cell proliferation contained $26 \mathrm{DE}$ genes that were primarily regulated by Sfpi1, Stat4, Tal1, Rel, Irf8, Stat1, Klf4 and Mybl2 DE TFs in the network. The regulators identified as the top five TFs involved in the positive regulation of $\mathrm{T}$ cell proliferation term, some of which have the same number of targets, for example Stat4 and Tal1 both regulate nine DE genes in this term. In addition to these processes, DE genes associated with tuberculosis were investigated based on KEGG pathway analysis of the gene regulatory network. A total of $38 \mathrm{DE}$ genes involved in tuberculosis were found to be present in the regulatory network constructed in the preset study. In addition, Sfpi1, Stat4, Tal1, Irf8, Klf4 and Stat1 were determined to be the top 5 DE TFs involved in regulation of Tuberculosis-associated DE genes in early lung immune response to tuberculosis. Overall, 4 DE TFs were identified (Sfpi1, Stat4, Tal1 and Klf4) which were actively involved in the regulation of the following three processes: Positive regulation of $\mathrm{T}$ cell activation, positive regulation of $\mathrm{T}$ cell proliferation and tuberculosis-associated gene expression.

In conclusion, according to the results of the analyses of TF involvement in protein complexes and active modules, centrality and the regulation of most enriched processes, it was revealed that the top DE TFs involved in the regulation of the early lung response to tuberculosis were Stat4, Irf8, Sfpi1, Ezh2 and Klf4.

\section{Discussion}

The present study identified possible novel TFs involved in the regulation of early lung immune response to tuberculosis in mice and their roles. Numerous TFs and their regulated processes in this constructed regulatory network were revealed to overlap with known regulators and affected processes during tuberculosis.

Stat4 has a crucial role in the development of the protective response against $M$. tuberculosis infection in mice (6). $\mathrm{CD}^{+}{ }^{+} \mathrm{T}$ cells deficient for Stat4 were reported to be unable to differentiate into Th1 cells during tuberculosis $(29,30)$. T cell receptors (TCR) induce activation of $\mathrm{CD}^{+} \mathrm{T}$ cells when they encounter antigens presented by antigen presenting cells (29). Activated T cells produce interleukin (IL)-2 and express IL-12 receptors in response to this signal. IL-12 receptors are activated on encountering IL-12, which is produced and secreted by macrophages and $\mathrm{CD} 8 \alpha^{+}$dendritic cells; in addition, the activated IL-12 receptor subsequently activates Stat4, which results in the initiation of Th1 differentiation from $\mathrm{CD}^{+}{ }^{+} \mathrm{T}$ cells during M. tuberculosis infection (29). During tuberculosis infection, Stat4 upregulation following IL-12 stimulation in bronchoalveolar cells was reported to lead to increased expression of interferon $\gamma(\operatorname{Inf} \gamma)(31)$.

Previous studies have suggested that Irf8 was critical for the differentiation of myeloid cells and defense against intracellular microbes $(8,10)$. A study by Marquis et al $(8)$ reported that uncontrolled M.tuberculosis growth occurred in the spleen, liver and lungs of BXH2 mice with a defective $I R F 8^{R 294 c}$ allele and induced premature mortality (8). Irf8 is a crucial regulator of the immune responses mediated by Th1 cells and is involved in regulation of toll-like signaling (32). A previous study reported that high overlap between the binding sites of Irf 8 and Spfil was observed in tuberculosis infection, as determined using ChIP-chip data analysis (10). Sfpil was demonstrated to be involved in the development of mature macrophages as well as B and T cells (33). This regulator primarily affected the efficiency of $\mathrm{T}$ cell progenitors fate commitment and/or their differentiation (34). In addition Sfpi1 was reported to have a role in the generation of cytokine expression patterns in T helper 2-type (Th2) cells (35).

Ezh2 is a methyltransferase component of polycomb repressive complex (PRC2) (36). Low expression of Ezh2 was reported in mature T cells; however, following antigen recognition through the TCR and activation of T cells Ezh2 expression was rapidly increased (36). Expression of cytokines during the development of Th1 and Th2 cells was predominantly regulated by PRC2 members (37). For example, Inf $\gamma$ expression was downregulated in Th1 cells following Ezh2 mRNA knock-down (37). IL-4 and IL-13 are Th2-specific cytokines, the expression levels of which were reported to be downregulated in Th1 cells through the methyltransferase activity of Ezh2 (38,39).

Mice with T cell knock-out of the Klf4 gene were used to investigate its roles in the development and differentiation of $\mathrm{T}$ cell. Klf4 was reported to be highly expressed in mature $\mathrm{T}$ cells; however, it was demonstrated that upon $\mathrm{T}$ cell activation, Klf4 expression was downregulated (40). Another study on the T cell activation network revealed the role of Klf4 in the regulation of transcription in tuberculosis (41).

Collectively, the present study aimed to identify novel TFs and dissect their roles in tge concept of gene regulatory network during early lung immune response to tuberculosis. This analysis led to identification of $17 \mathrm{DE}$ TFs involved in regulation of numerous immunological and biological processes, including $\mathrm{T}$ cell activation, $\mathrm{T}$ cell proliferation and tuberculosis-associated gene expression. Constructed network analysis revealed Stat4, Irf8, Sfpi1, Ezh2 and Klf4 as master regulators of early lung response to tuberculosis. Identification of these master TFs extend the current understanding of the underlying molecular mechanisms and may be useful as candidate novel targets for novel tuberculosis therapies.

\section{References}

1. World Health Organisation: Tuberculosis Fact Sheet. World Health Organization. Geneva Switzerland, 2012.

2. Leveton C, Barnass S, Champion B, Lucas S, De Souza B Nicol M, Banerjee D and Rook G: T-cell-mediated protection of mice against virulent Mycobacterium tuberculosis. Infect Immun 57: 390-395, 1989.

3. Kaufmann SH: Immunity to intracellular bacteria. Annu Rev Immunol 11: 129-163, 1993.

4. Schluger NW and Rom WN: The host immune response to tuberculosis. Am J Respir Crit Care Med 157 (3 Pt 1): 679-691, 1998.

5. Mogues T, Goodrich ME, Ryan L, LaCourse R and North RJ: The relative importance of $\mathrm{T}$ cell subsets in immunity and immunopathology of airborne Mycobacterium tuberculosis infection in mice. J Exp Med 193: 271-280, 2001.

6. Sugawara I, Yamada H and Mizuno S: Relative importance of STAT4 in murine tuberculosis. J Med Microbiol 52: 29-34, 2003.

7. Via LE, Tsytsykova AV, Rajsbaum R, Falvo JV and Goldfeld AE: The transcription factor NFATp plays a key role in susceptibility to TB in mice. PLoS One 7: e41427, 2012. 
8. Marquis JF, LaCourse R, Ryan L, North RJ and Gros P: Disseminated and rapidly fatal tuberculosis in mice bearing a defective allele at IFN regulatory factor 8 . J Immunol 182: 3008-3015, 2009

9. Keller C, Hoffmann R, Lang R, Brandau S, Hermann C and Ehlers S: Genetically determined susceptibility to tuberculosis in mice causally involves accelerated and enhanced and recruitment of granulocytes. Infect Immun 74: 4295-4309, 2006

10. Marquis JF, Kapoustina O, Langlais D, Ruddy R, Dufour CR Kim BH, MacMicking JD, Giguère V and Gros P: Interferon regulatory factor 8 regulates pathways for antigen presentation in myeloid cells and during tuberculosis. PLoS Genet 7: e1002097, 2011.

11. Corn RA, Hunter C, Liou HC, Siebenlist U and Boothby MR Opposing roles for RelB and $\mathrm{Bcl}-3$ in regulation of T-box expressed in T cells, GATA-3 and Th effector differentiation. J Immunol 175: 2102-2110, 2005.

12. Szabo SJ, Sullivan BM, Peng SL and Glimcher LH: Molecular mechanisms regulating Th1 immune responses. Annu Rev Immunol 21: 713-758, 2003.

13. Wang $\mathrm{X}$, Wang $\mathrm{H}$ and Xie J: Genes and regulatory networks involved in persistence of Mycobacterium tuberculosis. Sci China Life Sci 54: 300-310, 2011.

14. Sambarey A, Prashanthi K and Chandra N: Mining large-scale response networks reveals 'topmost activities' in Mycobacterium tuberculosis infection. Sci Rep 3: 2302, 2013.

15. Kumar D, Nath L, Kamal MA, Varshney A, Jain A, Singh S and Rao KV: Genome-wide analysis of the host intracellular network that regulates survival of Mycobacterium tuberculosis. Cell 140: 731-743, 2010.

16. Kang DD, Lin Y, Moreno JR, Randall TD and Khader SA: Profiling early lung immune responses in the mouse model of tuberculosis. PLoS One 6: e16161, 2011.

17. Irizarry RA, Hobbs B, Collin F, Beazer-Barclay YD, Antonellis KJ, Scherf U and Speed TP: Exploration, normalization and summaries of high density oligonucleotide array probe level data. Biostatistics 4: 249-264, 2003.

18. Blazejczyk M, Miron M and Nadon R: FlexArray: Statistical data analysis software for gene expression microarrays, made with life scientists in mind. McGill University and Génome Québec Innovation Centre, Montréal, QC, 2007.

19. Huang da W, Sherman BT and Lempicki RA: Systematic and integrative analysis of large gene lists using DAVID bioinformatics resources. Nat Protoc 4: 44-57, 2009.

20. Lachmann A, Xu H, Krishnan J, Berger SI, Mazloom AR and Ma'ayan A: ChEA: transcription factor regulation inferred from integrating genome-wide ChIP-X experiments. Bioinformatics 26 : 2438-2444, 2010.

21. Essaghir A, Toffalini F, Knoops L, Kallin A, van Helden J and Demoulin JB: Transcription factor regulation can be accurately predicted from the presence of target gene signatures in microarray gene expression data. Nucleic Acids Res 38: e120, 2010.

22. Stark C, Breitkreutz BJ, Reguly T, Boucher L, Breitkreutz A and Tyers M: BioGRID: a general repository for interaction datasets. Nucleic Acids Res 34 (Database Issue): D535-539, 2006.

23. Franceschini A, Szklarczyk D, Frankild S, Kuhn M, Simonovic M, Roth A, Lin J, Minguez P, Bork P, von Mering C and Jensen LJ: STRING v9.1: protein-protein interaction networks, with increased coverage and integration. Nucleic Acids Res 41 (Database Issue): D808-D815, 2013.

24. Saito R, Smoot ME, Ono K, Ruscheinski J, Wang PL, Lotia S, Pico AR, Bader GD and Ideker T: A travel guide to Cytoscape plugins. Nat Methods 9: 1069-1076, 2012.
25. Bindea G, Mlcenik B, Hackl H, Charoentong P, Tosolini M, Kirilovsky A, Fridman WH, Pagès F, Trajanoski $\mathrm{Z}$ and Galon J: ClueGO: a Cytoscape plug-in to decipher functionally grouped gene ontology and pathway annotation networks. Bioinformatics 25: 1091-1093, 2009.

26. Scardoni G, Petterlini M and Laudanna C: Analyzing biological network parameters with CentiScaPe. Bioinformatics 25: 2857-2859, 2009.

27. Bader GD and Hogue CW: An automated method for finding molecular complexes in large protein interaction networks. BMC Bioinformatics 4: 2, 2003.

28. Ideker T, Ozier O, Schwikowski B and Siegel AF: Discovering regulatory and signalling circuits in molecular interaction networks. Bioinformatics 18 (Suppl 1): 233-240, 2002.

29. Rengarajan J, Szabo SJ and Glimcher LH: Transcriptional regulation of Th1/Th2 polarization. Immunol Today 21: 479-483, 2000.

30. Wurster AL, Tanaka T and Grusby MJ: The biology of Stat 4 and Stat6. Oncogene 19: 2577-2584, 2000.

31. Raju B, Hoshino Y, Belitskaya-Lévy I, Dawson R, Ress S, Gold JA, Condos R, Pine R, Brown S, Nolan A, et al: Gene expression profiles of bronchoalveolar cells in pulmonary TB. Tuberculosis (Edinb) 88: 39-51, 2008.

32. Zhao J, Kong HJ, Li H, Huang B, Yang M, Zhu C, Bogunovic M, Zheng F, Mayer L, Ozato K, et al: IRF-8/interferon (IFN) consensus sequence-binding protein is involved in Toll-like receptor (TLR) signaling and contributes to the cross-talk between TLR and IFN-gamma signaling pathways. J Biol Chem 281: 10073-10080, 2006.

33. McKercher SR, Torbett BE, Anderson KL, Henkel GW, Vestal DJ, Baribault H, Klemsz M, Feeney AJ, Wu GE, Paige CJ and Maki RA: Targeted disruption of the PU.1 gene results in multiple hematopoietic abnormalities. EMBO J 15: 5647-5658, 1996.

34. Spain LM, Guerriero A, Kunjibettu S and Scott EW: T cell development in PU.1-deficient mice. J Immunol 163: 2681-2687, 1999.

35. Chang HC, Han L, Jabeen R, Carotta S, Nutt SL and Kaplan MH: PU.1 regulates TCR expression by modulating GATA-3 activity. J Immunol 183: 4887-4894, 2009.

36. He S, Tong Q, Bishop DK and Zhang Y: Histone methyltransferase and histone methylation in inflammatory T-cell responses. Immunotherapy 5: 989-1004, 2013.

37. Jacob E, Hod-Dvorai R, Ben-Mordechai OL, Boyko Y and Avni O: Dual function of polycomb group proteins in differentiated murine T helper (CD4+) cells. J Mol Signal 6: 5, 2011.

38. Koyanagi M, Baguet A, Martens J, Margueron R, Jenuwein T and Bix M: EZH2 and histone 3 trimethyl lysine 27 associated with II4 and II13 gene silencing in Th1 cells. J Biol Chem 280: 31470-31477, 2005

39. Lee GR, Kim ST, Spilianakis CG, Fields PE and Flavell RA: $\mathrm{T}$ helper cell differentiation: regulation by cis elements and epigenetics. Immunity 24: 369-379, 2006.

40. An J, Golech S, Klaewsongkram J, Zhang Y, Subedi K, Huston GE, Wood WH III, Wersto RP, Becker KG, Swain SL and Weng N: Krüppel-like factor 4 (KLF4) directly regulates proliferation in thymocyte development and IL-17 expression during Th17 differentiation. FASEB J 25: 3634-3645, 2011.

41. Subbian S, O'Brien P, Kushner NL, Yang G, Tsenova L, Peixoto B, Bandyopadhyay N, Bader JS, Karakousis PC, Fallows D and Kaplan G: Molecular immunologic correlates of spontaneous latency in a rabbit model of pulmonary tuberculosis. Cell Commun Signal 11: 16, 2013. 